Ann. Génét. Sél. anim., I973, 5 (3), 4I7-4I8.

\title{
ACTIVITY TEST ON YOUNG PHEASANTS (1)
}

\author{
J.-P. BOYER, J.-M. MELIN et P. BOURDENS \\ Station de Recherches avicoles, \\ Centre de Recherches de Tours, I. N. R. A., \\ Nouzilly B. P. 1, 37380 Monnaie
}

\section{SUMMARY}

Activity tests on young pheasants show a strong genetic component for this trait.

In view of a cynegetic repopulation, we have examined the activity of young pheasants, three/four days old by the mean of an open-field test. In june r 970 we have selected 40 dams and ro sires, half of them were classified as active (A) or inactive (I) ones, from individual tests.

The offspring of the two types has been brooded separately from the time of hatching and each young pheasant was examined, in an open-field equipped with 6 photo-electric cells, for " $t+\mathrm{r} 50$ seconds " $t$ being the latence time before first " top ".

The difference between the two offsprings seems very clear and gives the following results (table I).

Concerning the heritability of top-number, we obtain very high estimation when the two groups are pooled (table 2).

But when we take only A's offspring or I's offspring, the figure is consistently different (table 3).

In short, the results show a strong genetic component of behaviora lactivity. It has to be related with later characters such as aggressiveness, brooding, territoriality. The data at our hand seem to indicate the presence of such cynegetic qualities in "inactive " animals. It would be better to say " social and territorial " instead of "active or inactive " behavior.

If inactivity equals wildness, one can wonder, from our results : "Is wildness not governed by one major recessive gene?"

(1) Cet article a été présenté à la réunion du groupe de travail no 3 (sélection et testage) de la Fédération des Branches Européennes de la W.P.S.A., Nouzilly - Ploufragan, 6-ro septembre r97r. 
TABLE I. - Numbers of " tops" for progeny of "active " or " inactive " pheasants Nombres de tops pour les descendants de faisans "actifs " ou " inactifs"

\begin{tabular}{|c|c|c|c|c|c|c|c|c|}
\hline Mates & \multicolumn{4}{|c|}{ A (tops $200 \mathrm{~s}=43,0$ ) } & \multicolumn{4}{|c|}{ I (tops $200 \mathrm{~s}=7,7$ ) } \\
\hline Offspring & \multicolumn{2}{|c|}{$A \geqslant(14$ tops $)$} & \multicolumn{2}{|c|}{$\mathrm{I}(<14)$} & \multicolumn{2}{|c|}{$A(\geqslant 14)$} & \multicolumn{2}{|c|}{$I(<4)$} \\
\hline & $n$ & tops & $n$ & tops & $n$ & tops & $n$ & tops \\
\hline Hatch I & 33 & 33.4 & 58 & 5.2 & 12 & 23.8 & 95 & 3.7 \\
\hline Hatch II $\ldots$. & 39 & 26.2 & 70 & 5.2 & 6 & 29.8 & 101 & 2.7 \\
\hline All $(I+I I) \ldots$ & 72 & 29.5 & 128 & 5.2 & 18 & 25.8 & 196 & 3.2 \\
\hline$(I+I I) \%$ & \multicolumn{2}{|c|}{$A=36$} & \multicolumn{2}{|c|}{$I=64$} & \multicolumn{2}{|c|}{$\mathrm{A}=8.4$} & \multicolumn{2}{|c|}{$I=91.6$} \\
\hline Mean tops & \multicolumn{2}{|c|}{$\mathrm{A}=29.5$} & \multicolumn{2}{|c|}{$I=5.2$} & \multicolumn{2}{|c|}{$\mathrm{A}=25.8$} & \multicolumn{2}{|c|}{$I=3.2$} \\
\hline \multirow[t]{2}{*}{ Group mean } & \multicolumn{4}{|c|}{ A's offsp. $13.98 \pm 1,12$} & \multicolumn{4}{|c|}{ I's offsp. $5.06 \pm 0.58$} \\
\hline & \multicolumn{8}{|c|}{ Student's $t=7.19\left(\mathrm{P}<10^{-5}\right)$} \\
\hline
\end{tabular}

TABLE 2. - Heritability of top- numbers (all progeny) Héritabilité du nombre de tops (tous les descendants groupés)

\begin{tabular}{c|c|c|c}
\hline \hline & & $\mathrm{N}$ & $h_{\mathrm{S}}^{2}$ \\
\hline & & & \\
\hline Hatch I & all & 198 & .97 \\
Hatch II & all & 216 & .51 \\
I + II & pedigreed & 213 & .80 \\
I + II & 1/2 pedigreed & 201 & .62 \\
\hline
\end{tabular}

TABLE 3. - Heritability of top-numbers (Separating progeny of A and I individuals) Héritabilité $d u$ nombre de tops (en séparant la descendance des "actifs "et des "inactifs"

\begin{tabular}{c|c|c|c|c}
\hline \hline & & $\mathrm{N}$ & $h_{\mathrm{S}}^{2}$ & $h_{\mathrm{DS}}^{2}$ \\
\hline & & & & \\
\hline I + II & A's offsp. pedig. & 105 & .32 & .02 \\
I + II & I's offsp. pedig. & 108 & -.01 & .22 \\
\hline \hline
\end{tabular}

Reçu pour publication en mars 1973.

\section{RÉSUMÉ}

GÉNÉTIQUE DU COMPORTEMENT :

RÉSULTATS D'UN TEST D'ACTIVITÉ-INACTIVITÉ CHEZ DES FAISANDEAUX

Des tests d'activité sur de jeunes faisandeaux montrent une forte composante génétique pour ce caractère. 